\title{
Detection of ionospheric perturbations associated with Japanese earthquakes on the basis of reception of LF transmitter signals on the satellite DEMETER
}

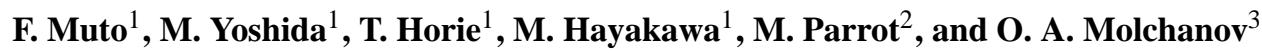 \\ ${ }^{1}$ Department of Electronic Engineering and Research Station on Seismo Electromagnetics, University of \\ Electro-Communications, Chofu Tokyo, Japan \\ ${ }^{2}$ LPCE/CNRS, Orleans, France \\ ${ }^{3}$ Institute of Physics of the Earth, Moscow, Russia
}

Received: 22 November 2007 - Revised: 22 January 2008 - Accepted: 22 January 2008 - Published: 26 February 2008

\begin{abstract}
There have been recently reported a lot of electromagnetic phenomena associated with earthquakes (EQs). Among these, the ground-based reception of subionospheric waves from VLF/LF transmitters, is recognized as a promising tool to investigate the ionospheric perturbations associated with EQs. This paper deals with the corresponding whistler-mode signals in the upper ionosphere from those VLF/LF transmitters, which is the counterpart of subionospheric signals. The whistler-mode VLF/LF transmitter signals are detected on board the French satellite, DEMETER launched on 29 June 2004. We have chosen several large Japanese EQs including the Miyagi-oki EQ (16 August 2005; $M=7.2$, depth=36 km), and the target transmitter is a Japanese LF transmitter (JJY) whose transmitter frequency is $40 \mathrm{kHz}$. Due to large longitudinal separation of each satellite orbit $(2500 \mathrm{~km})$, we have to adopt a statistical analysis over a rather long period (such as 3 weeks or one month) to have reliable data set. By analyzing the spatial distribution of JJY signal intensity (in the form of signal to noise ratio SNR) during a period of 4 months including the Miyagi-oki EQ, we have found significant changes in the intensity; generally the SNR is significantly depleted before the EQ, which is considered to be a precursory ionospheric signature of the EQ. This abnormal effect is reasonably explained in terms of either (1) enhanced absorption of whistler-mode LF signals in the lower ionosphere due to the lowering of the lower ionosphere, or (2) nonlinear wave-wave scattering. Finally, this analysis suggests an important role of satellite observation in the study of lithosphere-atmosphere-ionosphere coupling.
\end{abstract}

Correspondence to: M. Hayakawa

(hayakawa@whistler.ee.uec.ac.jp)

\section{Introduction}

EQ precursory signature is recently known to appear not only in the lithosphere, but also in the atmosphere and ionosphere (e.g., Hayakawa, 1999; Hayakawa and Molchanov, 2002). This means that EQs can excite atmospheric and ionospheric perturbations by direct coupling, which leads us to use a new terminology of "Lithosphere-AtmosphereIonosphere Coupling". The ionosphere seems to be disturbed in different height regions. For example, recent works by Liu et al. $(2000,2006)$ have suggested in the statistical sense that the ionospheric $\mathrm{F}$ layer is apparently disturbed during EQs. Also, we can cite a recent event study by Hobara and Parrot (2005). While, the lower ionosphere (D/E layer) is found already to be extremely sensitive to seismicity. This was confirmed by means of subionospheric VLF/LF propagation anomalies (Molchanov and Hayakawa, 1998) since the pioneering discovery of clear seismo-ionospheric perturbations for the Kobe EQ (Hayakawa et al., 1996). Because VLF/LF radio waves are known to propagate in the Earthionosphere waveguide, any change in the lower ionosphere may result in significant changes in the VLF signal received at a station (Molchanov and Hayakawa, 1998; Molchanov et al., 2001; Hayakawa, 2004; Hayakawa et al., 2004; Biagi et al., 2007). Recently, statistical analyses on the correlation between the lower ionospheric perturbation as detected by subionospheric VLF/LF signals and EQs, have been performed by Rozhnoi et al. (2004) and Maekawa et al. (2006), who have concluded that the lower ionosphere is definitely perturbed for the shallow EQs with magnitude greater than 6.0. Of course, it is not well understood at the moment how the ionosphere is perturbed due to the seismicity, though there have been proposed a few possible mechanisms on the lithosphere-atmosphere-ionosphere coupling

Published by Copernicus Publications on behalf of the European Geosciences Union. 


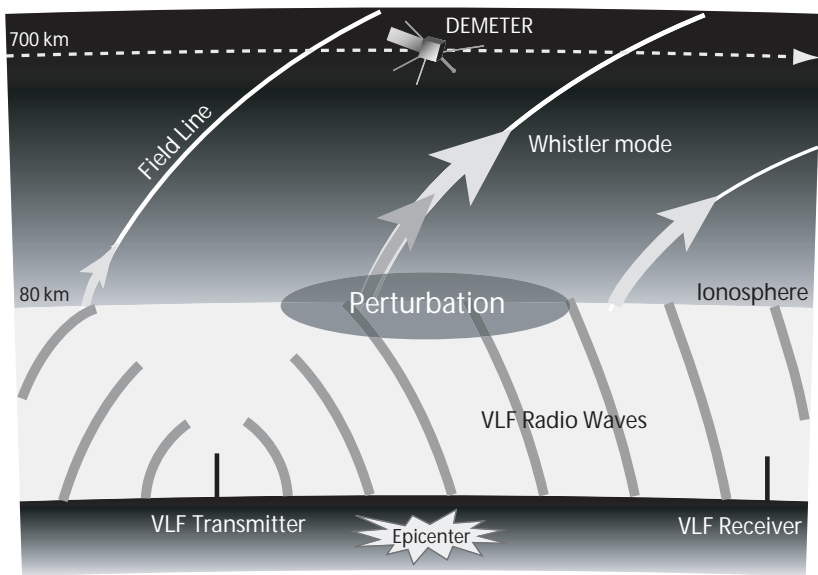

Fig. 1. The model of VLF/LF wave propagation, in which there are two modes of propagation: Earth-ionosphere waveguide mode and whistler mode in the ionospheric plasma. The mechanism of Lithosphere-Atmosphere-Ionosphere Coupling is plotted.

(see Molchanov et al., 2001; Hayakawa, 2004; Sorokin et al., 2005; Molchanov and Hayakawa, 2008).

As is shown in Fig. 1, a VLF/LF transmitter emits electromagnetic waves at a particular frequency mainly in the subionospheric waveguide, which are used to infer the seismo-ionospheric perturbations as mentioned above (e.g., Hayakawa et al., 1996; Molchanov and Hayakawa, 1998). While, another part of VLF/LF transmitter signals is known to penetrate upward into the ionosphere/magnetosphere in whistler mode (Hayakawa, 1995). This kind of whistlermode VLF transmitter signal is also expected to provide us with further information on the seismo-ionospheric perturbation because of their penetration through this region. In fact, Molchanov et al. (2006) have recently found significant seismo-ionospheric effects associated with a huge Sumatra EQ in December, 2004 by using the VLF data observed on board the French satellite, DEMETER. And, this satellite finding is found to be in good consistence with our ground-based VLF observation for the same EQ by Horie et al. (2007a, b). This paper is a further extension of the paper by Molchanov et al. (2006), which deals with further event studies for Japanese EQs by using the same DEMETER VLF/LF wave data. The satellite, DEMETER was launched on 29 June 2004, and it is working well with the aim of studying seismo-electromagnetic effects (Parrot et al., 2006). In this paper we have chosen several large Japanese EQs including the Miyagi-oki EQ (16 August 2005; M=7.2, depth $=36 \mathrm{~km}$ ), and the target transmitter is a Japanese LF transmitter (JJY) whose transmitter frequency is $40 \mathrm{kHz}$ and which is located in Fukushima prefecture (Hayakawa, 2004).

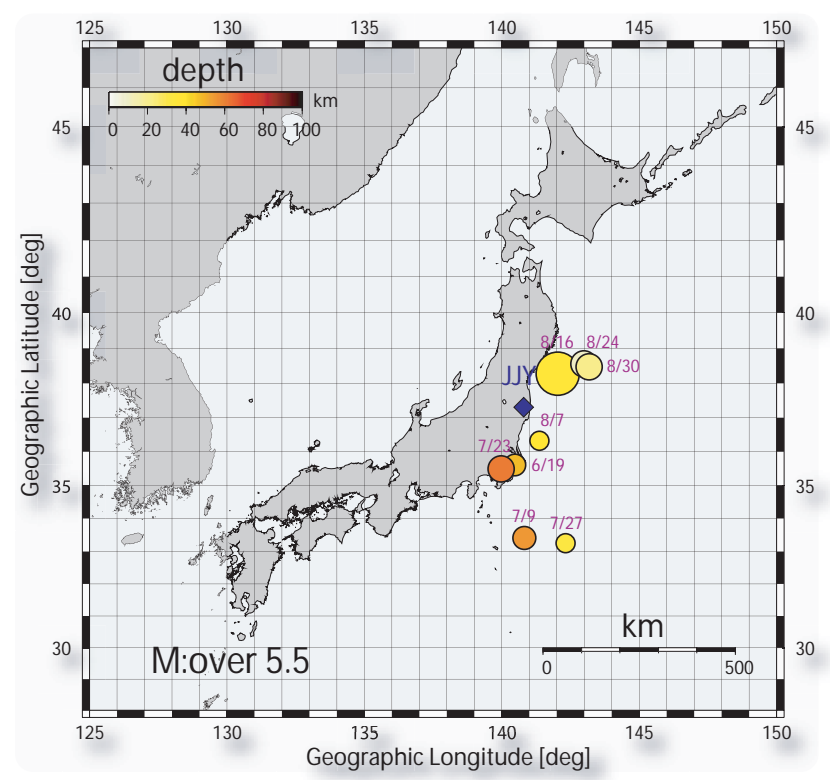

Fig. 2. Relative location of the LF transmitter in Fukushima, JJY and the epicenters of the target EQs including Miyagi-oki EQ. The size of an EQ is proportional to its magnitude.

\section{Analyzed EQs}

Figure 2 illustrates the relative location of our LF transmitter in Fukushima prefecture (geographic coordinates: $37^{\circ} 22^{\prime} \mathrm{N}$, $\left.140^{\circ} 51^{\prime} \mathrm{E}\right)$, and the epicenters of our target EQs around Japan. The JJY transmission frequency is $40 \mathrm{kHz}$, and the transmitter power is $50 \mathrm{~kW}$. These target EQs are summarized in Table 1, in which we have chosen several EQs during a period from 1 June 2005 to 30 September 2005. The selection criteria are as follows; (1) the magnitude of these EQs is greater than 5.5, and (2) the depth is less than $100 \mathrm{~km}$. Molchanov and Hayakawa (1998) indicated that only shallow EQs can influence the lower ionosphere, which is the reason why we adopt the latter condition The former criterion has been recently confirmed by statistical analyses by Rozhnoi et al. (2004) and Maekawa et al. (2006). As is seen in Table 1, we have a large EQ, named Miyagi-oki EQ happened on 16 August 2005 with magnitude of 7.2 and with depth of $36 \mathrm{~km}$. This EQ will be the main target of our analysis.

\section{LF wave data on DEMETER and analysis methods}

We follow the analysis method by Molchanov et al. (2006). Following Molchanov et al. (2006), we compute the signal to noise ratio (SNR) that is defined as follows.

$\mathrm{SNR}=\frac{2 \mathrm{~A}\left(\mathrm{f}_{0}\right)}{\mathrm{A}\left(\mathrm{f}_{-}\right)+\mathrm{A}\left(\mathrm{f}_{+}\right)}$. 
Table 1. Details of the EQs selected.

\begin{tabular}{llllll}
\hline Data & Lat. [deg] & Long. [deg] & Area & Depth $[\mathrm{km}]$ & M \\
\hline 2005.6 .19 & 35.61 & 140.48 & Chiba & 48 & 5.7 \\
2005.7 .9 & 33.42 & 140.82 & coast of southern Chiba & 55 & 5.8 \\
2005.7 .23 & 35.50 & 139.98 & Tokyo Bay & 61 & 6.1 \\
2005.7 .27 & 33.26 & 142.32 & coast of southeastern Chiba & 33 & 5.5 \\
2005.8 .7 & 36.33 & 141.37 & coast of Ibaraki & 39 & 5.5 \\
2005.8 .16 & 38.28 & 142.04 & coast of Miyagi & 36 & 7.2 \\
2005.8 .24 & 38.56 & 142.99 & coast of Miyagi & 10 & 6.1 \\
2005.8 .30 & 38.48 & 143.18 & coast of Miyagi & 21 & 6.1 \\
\hline
\end{tabular}

where $\mathrm{A}\left(\mathrm{f}_{0}\right)$ is the amplitude spectrum density in the frequency band including the transmitter (JJY) frequency $(40 \mathrm{kHz})$, and $\mathrm{A}\left(\mathrm{f}_{ \pm}\right)$are the background noise value outside the signal band. On the satellite DEMETER we observe the electromagnetic waves (electric field) in two different frequency bands: VLF range (below $20 \mathrm{kHz}$ ) and higher frequency range (above $20 \mathrm{kHz}$ ). Though the frequency resolution in the VLF range below $20 \mathrm{kHz}$ is sufficiently high $(19.53 \mathrm{~Hz})$, the corresponding resolution in the higher frequency range above $20 \mathrm{kHz}$ is not so high $(\Delta \mathrm{f}=3.255 \mathrm{kHz})$. So that we here describe how to choose the frequencies $\mathrm{f}_{0}$ and $\mathrm{f} \pm$, where $\mathrm{f}_{+}=\mathrm{f}_{0}+\Delta \mathrm{f}_{\text {and }} \mathrm{f}_{-}=\mathrm{f}_{0}-\Delta \mathrm{f}$. This $\Delta \mathrm{f}$ should be large enough as a necessary condition in order to avoid the spectral broadening due to the wavewave interaction between the transmitter signal and ionospheric turbulence as found by Bell et al. (1983), Titova et al. (1984) and Tanaka et al. (1987), the maximum value being $500 \mathrm{~Hz}$. Then, we have studied the electric field spectra over Japan, and Fig. 3 is the electric field spectrum averaged around Japan (latitude $=10^{\circ} \sim 50^{\circ}$, longitude $=110^{\circ} \sim 160^{\circ}$ ) during one month of August, 2005 in the sensitive area to be defined later. In the analysis we use only the nighttime data (in this analysis, just around L.T. $=22 \mathrm{~h}$ ) because of the lower attenuation in the ionospheric D/E region. By examing the electric field spectra like Fig. 3, we have chosen the relevant frequencies as $\mathrm{f}_{0}=39.1 \mathrm{kHz}, \mathrm{f}_{-}=32.6 \mathrm{kHz}$ and $\mathrm{f}_{+}=45.6 \mathrm{kHz}$ in the following estimation of SNRs. The use of SNR enables us to estimate the signal above the background more quantitatively than the simple intensity of $\mathrm{A}\left(\mathrm{f}_{0}\right)$.

Due to the trajectory problem of the DEMETER satellite, the separation in longitude of successive orbits is $2500 \mathrm{~km}$. This would suggest us to adopt any statistical treatment of the SNR data acquisition. Fig. 4 shows this situation. In Fig. 4a we plot the spatial distribution of SNR for one particular day (6 September 2005), in which the size of a circle along the orbit corresponds to the value of SNR. A larger circle corresponds to a higher SNR and a smaller circle, a lower SNR. Figure $4 \mathrm{~b}$ indicates the corresponding spatial distribution of SNRs, by increasing the integration time up to 3 weeks. That is, Fig. $3 b$ is the plot of SNRs for all orbits during 3 weeks

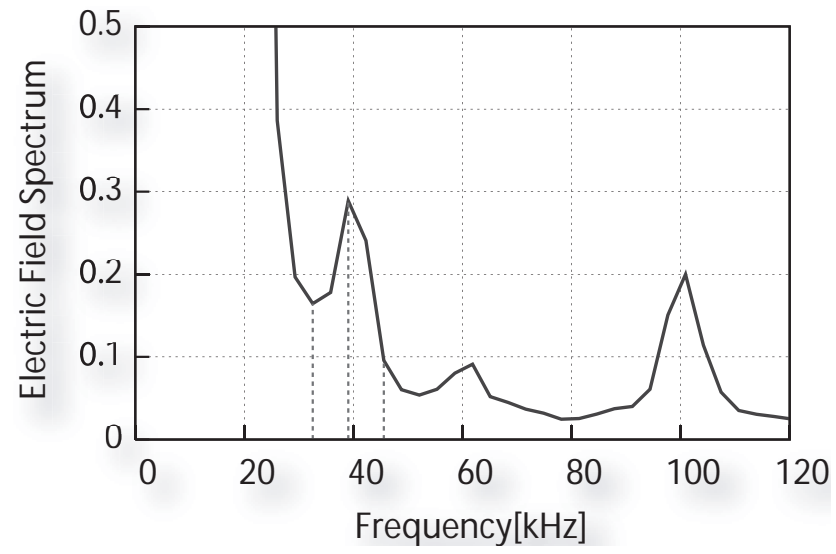

Fig. 3. A typical spectrum of electric field intensity (in linear scale). This spectrum is computed as the average of SNRs in a rectangular area (latitude: $10 \sim 50[\mathrm{deg}]$, longitude: $110 \sim 160[\mathrm{deg}])$. The three vertical dotted lines correspond to the frequencies $\left(f_{-}, f_{0}\right.$ and $\left.f_{+}\right)$.

(6 September to 26 September 2005) and this is described as the result for 26 September. This integration time of 3 weeks seems to be ready for the statistical analysis, so that we choose 3 weeks for the temporal integration analysis in the following presentations.

We here mention the normalization of the SNR data and how to choose the sensitive area. As is seen in Fig. 4c, we divide the whole analysis region in Fig. $4 \mathrm{~b}$ into many segments with a size of $2^{\circ}$ (in latitude) and $2^{\circ}$ (in longitude). This segment size is chosen in such a way to have sufficient spatial resolution, and to have significant number of orbits within it. We compute the average of the SNRs among adjacent 9 pixels and we regard this average as representing the central pixel. We repeat this procedure, and Fig. $4 \mathrm{~d}$ is the result based on such a normalization method. This figure indicates that there exists an area with enhanced SNRs mainly in the southern side of the transmitter. This is easily understood when we think of the characteristics of whistler-mode propagation at low latitudes that the whistler-mode signal propagates approximately along the Earth's magnetic field 


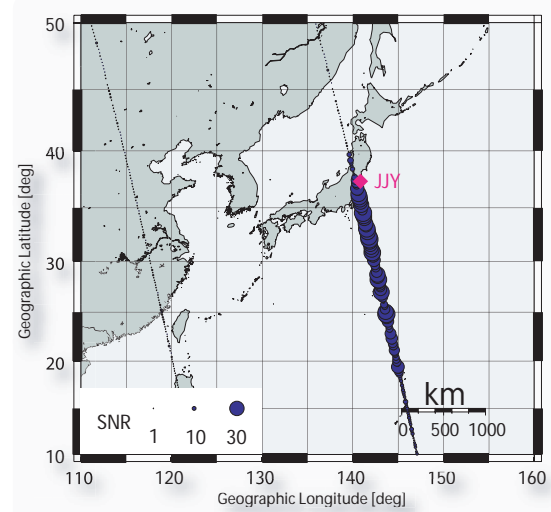

(a) SNR plots for 1 day

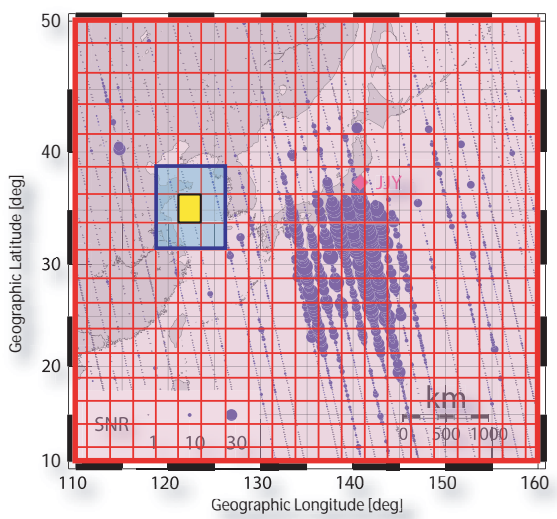

(c) Divide region into $2 \times 2[\mathrm{deg}]$

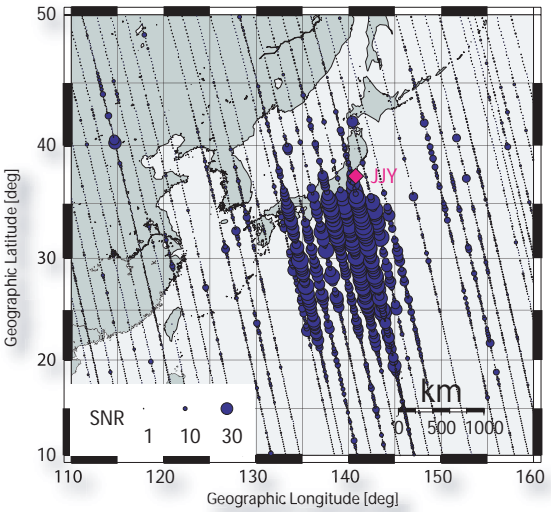

(b) SNR plots for 3 weeks

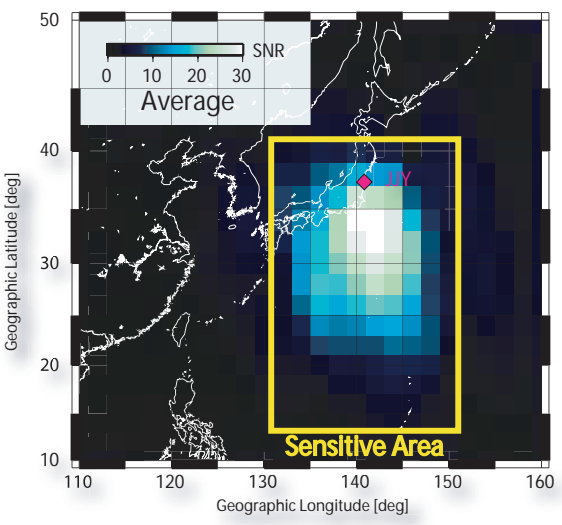

(d) Normalized SNR $(2 \times 2[\mathrm{deg}])$

Fig. 4. Analysis methods. (a) SNR plots for 1day, and (b) SNR plots for 3 weeks. (c) Diving the region into $2 \times 2$ [deg] pixels, and (d) normalized SNR. The color map indicates the value of SNR in each cell.

(Hayakawa, 1995). We tentatively define a yellow rectangular area in Fig. 4d as a "sensitive area" in our analysis.

Figure 5 illustrates the temporal evolution of the spatial distribution of SNRs for the largest EQ (Miyagi-oki EQ in August 2005). Those figures are obtained one week ( -7 days) before the EQ (Fig. 5a), on the EQ day (Fig. 5b), 10 days (Fig. 5c) and 20 days (Fig. 5d) after the EQ. A comparison of a series of these spatial distributions in Fig. 5, indicates that the region of high SNR values shrinks as the day of the Miyagi-oki EQ is approached. That is, we notice significant changes in Figs. 5a and 5b. Some changes are seen to take place in Fig. 5b (EQ day), which is based on the data during the period of 3 weeks before the EQ. And, Fig. 5c (+10 days; 10 days after the EQ) is based on the data during 3 weeks before +10 days (that is, about 2 weeks before the EQ and about one week after the EQ), which indicates a noticeable shrink of the high SNR region (i.e., decrease in SNR). While, the spatial distribution of SNRs in Fig. 5d (+20 days) is found to be close to Fig. $5 \mathrm{a}$, which means that the region of high SNRs is expanded and seems to have recovered to the background level.
Then we compute the average SNRs in the sensitive area in Fig. 5 to estimate the temporal variation of SNR. Figure 6 is the result on the temporal evolution of average SNR in the sensitive area during the whole period of our analysis, including the above-mentioned Miyagi-oki EQ. At the top of the figure, the times of EQs and their magnitude (downward) are plotted for the sake of comparison. The big EQ on 16 August 2005 is the Miyagi-oki EQ with magnitude of 7.2, and the subsequent two EQs on 24 August and $30 \mathrm{Au}-$ gust are its aftershocks because they occurred nearly at the same place as the main shock. A comparison between the EQ time and average SNR in the sensitive area, suggests the following important finding. There are present rapid variations (of the order of 1 day) and slow variations (of the scale of a week) in the temporal variation of SNR in Fig. 6. But, we are interested in these slow variations, so that we focus only on the slow variations especially with decreasing SNR, and Fig. 6 shows that with taking into account the integration time of 3 weeks, the average SNR in the sensitive area begins to decrease about a few weeks before the most intense Miyagi-oki EQ and such a decrease continues until the EQ. This decrease in SNR is indicated by a declined arrow. The 


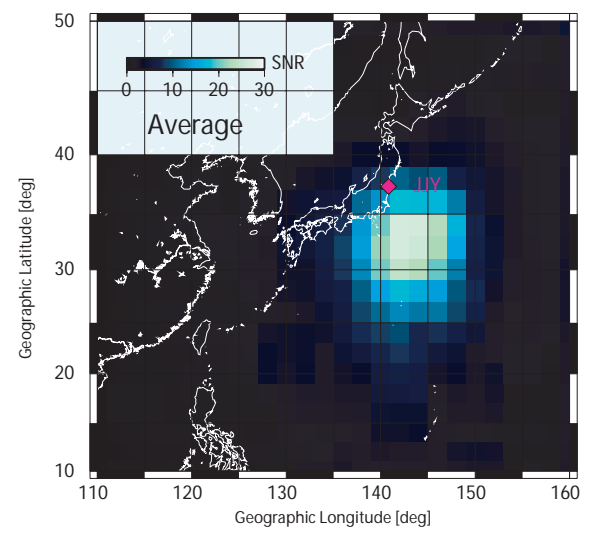

(a) 2005.8 .9 (-7 days)

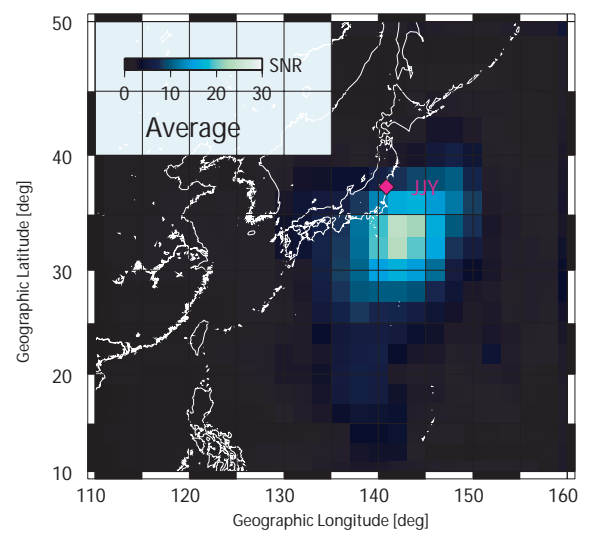

(c) 2005.8 .26 ( +10 days)

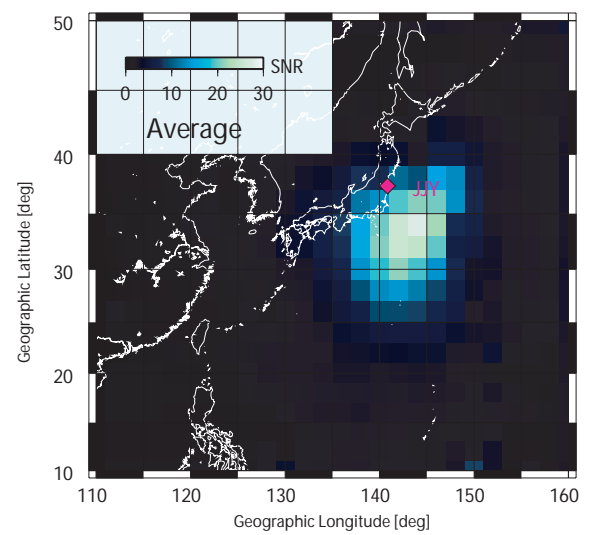

(b) 2005.8.16 ( Miyagi-Oki EQ occurrence)

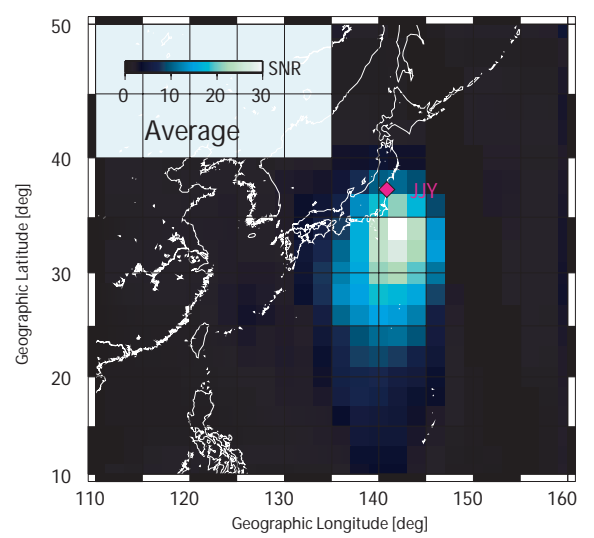

(d) 2005.9 .5 (+20 days)

Fig. 5. Temporal evolution of spatial SNR distributions. (a) 7 days before the EQ, (b) 0 day (EQ day), (c) 10 days after the EQ and (d) 20 days after the EQ.

decrease in SNR is about $20 \%$ in the average value, so that the instantaneous decrease in SNR must be much larger than this value. Then, the SNR value increases (or relaxes) from about 10 days after the EQ. As the general conclusion, the close correction between the decrease in SNR before this EQ and EQ occurrence, is very obvious. The magnitude of two aftershocks was both around 6.0, but their effect seems to be not so significant as seen in Fig. 6. Because the temporal evolution of average SNR in Fig. 6 after the Miyagi-oki EQ looks just a simple relaxation of this big EQ. This change is considered to support qualitatively the conclusion obtained in Fig. 5.

Next we look at other EQ events in the upper panel of Fig. 6. There are four extensive and isolated EQs before the Miyagi-oki EQ in August. It is not so difficult for us to find the similar behavior as for the Miyagi-oki EQ. That is, the decrease in SNR value starts about a few weeks before the EQ as shown by the same declined arrows. As summarizing the results for these EQs, it is concluded that any EQ is preceded always by the decrease in the SNR value in the sensitive area.

As is already statistically confirmed by Rozhnoi et al. (2004) and Maekawa et al. (2006), the seismic effect can be clearly seen for any EQs with magnitude greater than 5.56.0 , irrespective of other conditions including geomagnetic activity etc. The lower panel of Fig. 6 is the temporal evolution of geomagnetic activity expressed by Dst index, which indicated a relatively quiet geomagnetic activity during our analysis period except one magnetic storm in the end of $\mathrm{Au}-$ gust. Even this storm had no effect on the temporal evolution of SNR.

\section{Conclusions}

Based on the VLF/LF wave observation on board the French DEMETER satellite, of a Japanese standard transmitter, JJY we have investigated the ionospheric perturbations for a relatively large EQ (Miyagi-oki EQ on 16 August 2005) and also for several isolated EQs around Japan. The EQs selected have magnitude greater than 5.5 and depth less than $100 \mathrm{~km}$. First of all, the SNR signals in the ionosphere by DEMETER are found to be enhanced in the southern side of the transmitter, due to the intrinsic whistler-mode propagation characteristics. This sensitive area for the Miyagi-oki EQ is found to 

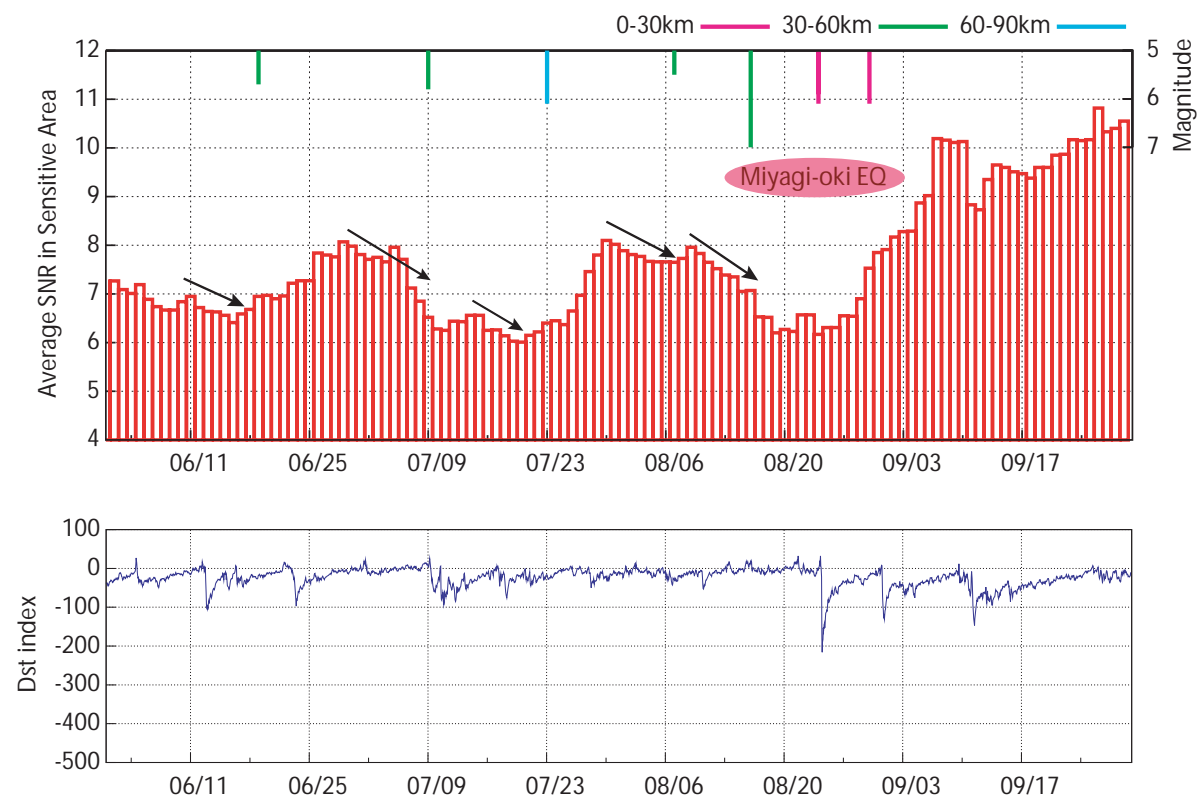

Fig. 6. Upper panel: The temporal variation of the average of SNR in the Sensitive Area. The declining arrow above the average SNR indicates the decrease in the average SNR in the sensitive area. While, the information on EQ is given at the top of the figure (magnitude in vertically downward bar, and depth is given in color). Lower panel: Temporal evolution of Dst index.

begin to shrink about a few weeks before the EQ, followed by the relaxation to the background level. This general property is found to be universal for other several EQs.

We could confirm the decrease in electric field intensity of the JJY transmitter signal in the ionosphere on board the DEMETER for several isolated EQs around Japan, which is in excellent agreement with our recent paper by Molchanov et al. (2006). Also, the temporal evolution of SNRs in our sensitive area seems to be again consistent with Molchanov et al. (2006)'s result, because both of these results indicate the precursory effect of EQs. However, in this paper we cannot infer any other information on the ionospheric perturbation, such as its spatial scale. Because Molchanov et al. (2006) have used a very powerful VLF transmitter like NWC in Australia, they have succeeded in estimating the scattering spot, such as the scale of the ionospheric perturbation being $\sim 5000 \mathrm{~km}$ in diameter for the huge Sumatra EQ. Unfortunately, our LF transmitter in this study, JJY is a rather weak transmitter, which made us unable to estimate such a dimension of the seismo-ionospheric perturbation. We lastly comment on the depths of all EQs treated in this paper (as seen in Table 1). The depth of all EQs are less than $60 \mathrm{~km}$ and all EQs are shallow. We have already confirmed that only shallow EQs have an effect onto the ionosphere (Molchanov and Hayakawa, 1998), and significant effects for all EQs in Fig. 6 are consistent with our previous finding.

This kind of phenomenon such as the depletion of whistler-mode LF transmitter signals detected within the ionosphere can be explained by a few possible mechanisms. The whistler-mode signal propagates through the lower iono- sphere, so that the perturbation in the lower ionosphere prior to an EQ (such as the lowering of the lower ionosphere as suggested by Hayakawa et al. 1996 and Molchanov and Hayakawa 1998) might result in the enhanced absorption in the lower ionosphere. This can explain the observational fact presented in this paper. Another possibility is the nonlinear wave-wave interaction suggested by Molchanov and Hayakawa (2008), and Molchanov et al. (2006). The initial agent might be an upward energy flux of atmospheric gravity waves which are induced by the gas-water release from the EQ preparatory zone (e.g., Molchanov and Hayakawa, 2008). The penetration of atmospheric gravity waves into the ionosphere leads to modification of the natural ionospheric turbulence (Molchanov et al., 2004; Hobara et al., 2005), and then resonant scattering of the VLF transmitter signal with these turbulences might be possible. We need further study to elucidate the mechanism in future.

Acknowledgements. The authors are grateful to J. J. Berthelier for his electric field measurement on DEMETER, and thanks are also due to NICT for its support ("R and D promotion scheme funding international joint research").

Edited by: M. Contadakis

Reviewed by: P. F. Biagi and another anonymous referee 


\section{References}

Bell, T. F., James, H. G., Inan, U. S., and Katsufrakis, J. P.: The apparent spectral broadening of VLF transmitter signals during transionospheric propagation, J. Geophys. Res., 88, 4813-4816, 1983.

Biagi, P. F., Castellana, L., Maggipinto, T., Maggipinto, G., Minafra, A., Ermini, A., Capozzi, V., Perna, G., Solovieva, M., Rozhnoi, A., Molchanov, O. A., and Hayakawa, M.: Decrease in the electric intensity of VLF/LF radio signals and possible connections, Nat. Hazards Earth Syst. Sci., 7, 423-430, 2007, http://www.nat-hazards-earth-syst-sci.net/7/423/2007/.

Hayakawa, M.: Atmospheric and Ionospheric Electromagnetic Phenomena Associated with Earthquakes, Terra Sci. Pub. Co., Tokyo, 996 pp., 1999.

Hayakawa, M.: Electromagnetic phenomena associated with earthquakes: A frontier in terrestrial electromagnetic noise environment, Recent Res. Devel. Geophysics, 6, 81-112, 2004.

Hayakawa, M. and Molchanov, O. A.: NASDA/UEC team, Summary report of NASDA's earthquake remote sensing frontier project, Phys. Chem. Earth, 29, 617-625, 2004.

Hayakawa, M., Molchanov, O. A., Ondoh, T., and Kawai, E.: The precursory signature effect of the Kobe earthquake on VLF subionospheric signals, J. Comm. Res. Lab., Tokyo, 43, 169$180,1996$.

Hayakawa, M.: Whistlers, Chapt. 7, in: Handbook of Atmospheric Electrodynamics, edited by: Volland, H., vol.II, CRC Press, 155193, 1995.

Hayakawa, M. and Molchanov, O. A.: Seismo Electromagnetics: Lithosphere-Atmosphere - Ionosphere Coupling, TERRAPUB, Tokyo, 477 pp., 2002.

Hobara, Y. and Parrot, M.: Ionospheric perturbations linked to a very powerful seismic event, J. Atmos. Solar-Terr. Phy., 67, 677685, 2005.

Hobara, Y., Lefeuvre, F., Parrot, M., and Molchanov, O. A.: Low latitude ionospheric turbulence observed by Aureol-3 satellite, Ann. Geophys., 23, 1259-1270, 2005,

http://www.ann-geophys.net/23/1259/2005/.

Horie, T., Maekawa, S., Yamauchi, T., and Hayakawa, M.: A possible effect of ionospheric perturbations associated with the Sumatra earthquake, as revealed from subionospheric very-lowfrequency (VLF) propagation (NWC-Japan), Int. J. Remote Sensing, 28, 13, 3133-3139, 2007a.

Horie, T., Yamauchi, T., Yoshida, M., and Hayakawa, M.: The wave-like structures of ionospheric perturbation associated with Sumatra earthquake of 26 December 2004, as revealed from VLF observation in Japan of NWC signals, J. Atmos. Solar-Terr. Phy., 69, 1021-1028, 2007b.

Liu, J. Y., Chen, Y. I., Pulinets, S. A., Tsai, Y. B., and Chuo, Y. J.: Seismo-ionospheric signatures prior to $\mathrm{M}>6.0$ Taiwan earthquakes, Geophys. Res. Lett., 27, 3113-3116, 2000.
Liu, J. Y., Chen, Y. I., Chuo, Y. J., and Chen, C. S.: A statistical investigation of preearthquake ionospheric anomaly, J. Geophys. Res., 111, A05304, doi:10.1029/2005JA011333, 2006.

Maekawa, S., Horie, T., Yamauchi, T., Sawaya, T., Ishikawa, M., Hayakawa, M., and Sasaki, H.: A statistical study on the effect of earthquakes on the ionosphere, based on the subionospheric LF propagation data in Japan, Ann. Geophys., 24, 2219-2225, 2006, http://www.ann-geophys.net/24/2219/2006/.

Miyaki, K., Hayakawa, M., and Molchanov, O. A.: The role of gravity waves in the lithosphere-ionosphere coupling, as revealed from the subionospheric LF propagation data, in: Seismo Electromagnetics: Lithosphere-Atmosphere-Ionosphere Coupling, edited by: Hayakawa, M. and Molchanov, O. A., TERRAPUB, Tokyo, 229-232, 2002.

Molchanov, O. A., Akentieva, O. S., Afonin, V. V., Mareev, E. A., and Fedorov, E. N.: Plasma density-electric field turbulence in the low-latitude ionosphere from the observation on satellites; possible connection with seismicity, Phys. Chem. Earth, 29, 569577, 2004.

Molchanov, O. A., Rozhnoi, A., Solovieva, M., Akentieva, O., Berthelier, J. J., Parrot, M., Lefeuvre, F., Biagi, P. F., Castellana, L., and Hayakawa, M.:Global diagnostics of the ionospheric perturbations related to the seismic activity using the VLF radio signals collected on the DEMETER satellite, Nat. Hazards Earth Syst. Sci., 6, 745-753, 2006, http://www.nat-hazards-earth-syst-sci.net/6/745/2006/.

Molchanov, O. A., Hayakawa, M., and Miyaki, K.: VLF/LF sounding of the lower ionosphere to study the role of atmospheric oscillations in the lithosphere-ionosphere coupling, Adv. Polar Upper Atmos. Res., 15, 146-158, 2001.

Molchanov, O. A. and Hayakawa, M.: Subionospheric VLF signal perturbations possibly related to earthquakes, J. Geophys. Res., 103, 17, 489-17, 504, 1998.

Molchanov, O. A. and Hayakawa, M.: Seismo-electromagnetics and Related Phenomena: History and Latest Results, TERRAPUB, in press, 2008.

Parrot, M.: First results of the DEMETER micro-satellite, Planet. Space Sci., 54, 5, 2006.

Rozhnoi, A., Solovieva, M. S., Molchanov, O. A., and Hayakawa, M.: Middle latitude LF $(40 \mathrm{kHz})$ phase variations associated with earthquakes for quiet and disturbed geomagnetic conditions, Phys. Chem. Earth, 29, 589-598, 2004.

Sorokin, V. M., Yaschenko, A. K., Chmyrev, V. M., and Hayakawa, M.: DC electric field amplification in the mid-latitude ionosphere over seismically active faults, Nat. Hazards Earth Syst. Sci., 5, 661-666, 2005, http://www.nat-hazards-earth-syst-sci.net/5/661/2005/. 